\title{
Desafios da saúde pública no Brasil: relação entre zoonoses e saneamento
}

A saúde no Brasil deu início no período colonial a partir do século XIX, porém o Ministério da Saúde somente foi construído em 25 de julho de 1953 com a lei no 1.920. Desde ano de 1950 até o final do século passado, o investimento em saneamento básico no Brasil ocorreu pontualmente em alguns períodos específicos. O objetivo do presente trabalho é gerar uma discussão acerca da relação entre as zoonoses mais frequentes no país e o saneamento dando ênfase também nas medidas preventivas de doenças emergentes e re-emergentes no território nacional. As doenças zoonóticas são aquelas transmitidas dos animais para os humanos, as mais conhecidas são Doença de chagas, Raiva, Leishmanioses, Leptospirose, Febre Amarela, Dengue e Malária. As ações humanas sobre o ambiente como a urbanização, pecuária e industrialização vêm proporcionando um maior contato entre seres humanos, animais silvestres e domésticos, facilitando assim a proliferação de agentes infecciosos. Nos últimos anos, tem-se notado episódios de novas enfermidades bem como o reaparecimento de doenças datadas como erradicadas. Diante de todo trabalho realizado acrescentou-se medidas para a melhoria e prevenção de algumas zoonoses, a importância dos serviços de saneamento básico na prevenção de doenças e ações na preservação do ambiente.

Palavras-chave: Saneamento; Saúde Pública; Zoonoses.

\section{Challenges of public health in Brazil: relationship between zoonoses and sanitation}

\begin{abstract}
Health in Brazil began in the colonial period from the nineteenth century, but the Ministry of Health was only built on July 25, 1953 with the law n.1920. From the 1950s to the end of the last century, investment in basic sanitation in Brazil took place on a few specific periods. The objective of the present work is to generate a discussion about the relationship between the most frequent zoonoses in the country and the sanitation, emphasizing also the preventive measures of emerging and re-emergent diseases in the national territory. Zoonotic diseases are those transmitted from animals to humans, the most known of which are Chagas disease, Rabies, Leishmaniasis, Leptospirosis, Yellow Fever, Dengue and Malaria. Human actions on the environment such as urbanization, livestock and industrialization have provided greater contact between humans, wild and domestic animals, thus facilitating the proliferation of infectious agents. In recent years, there have been episodes of new illnesses as well as the reappearance of diseases dating as eradicated. In the light of all the work done, there were added measures for the improvement and prevention of some zoonoses, the importance of basic sanitation services in the prevention of diseases and actions in the preservation of the environment.
\end{abstract}

Keywords: Sanitation; Public Health; Zoonoses.

Topic: Políticas, Planejamento e Gestão de Sistemas e Serviços de Saúde

Reviewed anonymously in the process of blind peer.

Cristianne Ferreira Machado Rodrigues

Faculdade Guaraí, Brasil

http://lattes.cnpq.br/6688597592329908

cristiannefmachado@hotmail.com

Valdeir Santos Rodrigues

Faculdade Guaraí, Brasil

http://lattes.cnpq.br/0598005606753381

valdeir.srodrigues@hotmail.com

Júlio Cesar Ibiapina Neres (iD

Pontifícia Universidade Católica de Goiás, Brasil

http://lattes.cnpq.br/9493252013144748

http://orcid.org/0000-0002-5277-0790

biologia@faculdadeguarai.com.br

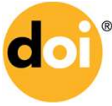

DOI: 10.6008/SPC2236-9600.2017.001.0003
Received: 09/01/2017

Approved: 20/07/2017

\begin{abstract}
Ana Paula Martins Guimarães
Universidade Federal do Tocantins, Brasil http://lattes.cnpq.br/5309168123830683 biologa.apmg@gmail.com

Liberta Lamarta Favoritto Garcia Neres

Universidade Brasil, Brasil

http://lattes.cnpq.br/0563097731032602

http://orcid.org/0000-0003-1327-605X

libertalamarta@gmail.com

Aluísio Vasconcelos Carvalho iD

Universidade Federal do Tocantins, Brasil http://lattes.cnpq.br/5200758055263996 http://orcid.org/0000-0002-3793-3133

aluisiovasconcelos@gmail.com
\end{abstract}

\section{Referencing this:}

RODRIGUES, C. F. M.; RODRIGUES, V. S.; NERES, J. C. I.; GUIMARÃES, A. P. M.; NERES, L. L. F. G.; CARVALHO, A. V.. Desafios da saúde pública no Brasil: relação entre zoonoses e saneamento. Scire Salutis, v.7, n.1, p.27-37, 2017. DOI: http://doi.org/10.6008/SPC22369600.2017.001.0003 


\section{INTRODUÇÃO}

A saúde no Brasil deu início no período colonial, a partir do século XIX, entretanto a assistência à saúde trouxe maior influência com a instrução da prática médica e com a criação de hospitais públicos para acolher doentes que necessitaria de maiores cuidados (RIBEIRO et al., 2010).

O Ministério da Saúde foi construído em 25 de julho de 1953 com a lei no 1.920 que criando também o de educação (LIMA \& PINTO, 2003). No ano de 1956 com o decreto 2.743 foi criado o setor de endemias rurais (DNERu), tinha como finalidade investigar e executar o serviço de combate à malária, leishmaniose, doenças de chagas, peste, brucelose, febre amarela, esquistossomose, ancilostomose, filariose, hidatidose, bócio endêmico, bouba, tracoma e outras endemias existentes no país (SILVEIRA \& PIMENTA JUNIOR, 2011).

A partir de 1950 até o final do século passado, o investimento em saneamento básico no Brasil ocorreu pontualmente em alguns períodos específicos, com um destaque para os anos de 1970 e 1980 tornando prioridade o atendimento a demandas de coleta e tratamento de esgoto (LEONETI et al., 2011). A ausência de saneamento e o não cuidado com a higiene pessoal da população podem ser considerados como fatores de risco à saúde pública (SOARES et al., 2002).

A atuação do saneamento básico promove o avanço da qualidade de vida da população refletindo positivamente na saúde pública com diminuição da mortalidade infantil, além da redução de doenças (TEIXEIRA \& GUILHERMINO, 2006).

A importância do saneamento básico para a saúde pública preconiza medidas preventivas para o combate a doenças, sua ausência ou existência precária constitui um fator limitante à promoção de água potável por parte do ser humano, além disso, beneficia a manifestação de patologias como diarréia, Febres entéricas, Hepatite A, Dengue, Febre Amarela, Leishmanioses, Filariose linfática, Malária, Doença de Chagas, Esquistossomose, Leptospirose, Doenças dos olhos, Tracoma, Conjuntivites, Doenças da pele, Micoses superficiais, Helmintíases, Teníases, cólera, febre tifóide e paratifóide, doenças intestinais, entre outras doenças emergentes e re-emergentes no país (FRANCEYS et al., 1994; OMS, 2017).

Os riscos à saúde pública podem estar ligado a fatores indesejados em áreas urbanas e rurais e que devem ser eliminadas com o uso adequado do serviço de saneamento, com a utilização de água potável e sistemas de esgoto, evitando a proliferação de vetores (BRASIL, 2011a).

Mais de um bilhão de habitantes na Terra não têm acesso à habitação segura e a serviços básicos de saúde, apesar de todas as pessoas terem direito a uma vida saudável e produtiva, em harmonia com o ambiente em que vive. No Brasil, as doenças resultantes da falta ou de um inadequado sistema de saneamento, principalmente em áreas pobres e vulneráveis, têm agravado o quadro epidemiológico no país (BRASIL, 2006a).

Tendo em vista a problemática do estudo, o objetivo do presente trabalho é gerar uma discussão acerca da relação entre as zoonoses mais frequentes no país e o saneamento brasileiro dando ênfase também nas medidas preventivas de doenças emergentes e re-emergentes no território nacional. 


\section{METODOLOGIA}

Trata de um estudo de revisão teórica, que tem como objetivo principal aprimorar ideias ou descobertas, envolvendo levantamento bibliográfico (GIL, 2002). Para o levantamento de artigos, utilizou-se as palavras-chave "saneamento básico", "saúde pública", "educação ambiental", "educação em saúde" e "zoonoses" publicados em plataformas de periódicos Sientific Electronic Library Online (SciELO), Google Acadêmico, Coordenação de Aperfeiçoamento de Pessoal de Nível Superior (CAPES) e LILACS (Literatura Latino-Americana em Ciências da Saúde) nos últimos dez anos.

\section{DISCUSSÃO TEÓRICA}

\section{Doenças Zoonóticas e sua Prevalência no Brasil}

As doenças zoonóticas são aquelas transmitidas dos animais para o ser humano, estão espalhadas no mundo de acordo com as diversas alterações ambientais, socioeconômicas e culturais. Os casos vêm aumentando gradualmente devido às constantes modificações causadas pelo o homem no ambiente, na maioria das vezes, os casos de doenças estão em regiões onde as populações são carentes, possui hábitos precários de higiene e baixa renda, propiciando um espaço maior para a infecção por agentes patogênicos (LIMA et al., 2017).

As doenças zoonóticas mais conhecidas são doenças de chagas, raiva, leishmanioses, leptospirose, febre amarela, dengue e malária, transmitidas por vetores que convivem com humanos através de sinantropia (SANGUINETTE, 2015).

A Doença de Chagas é uma antropozoonose ocasionada pelo protozoário flagelado Trypanosoma cruzi. A doença tem duas fases: uma crônica e aguda. Já no Brasil, devido à transmissão vetorial, predominam os casos crônicos decorrentes de infecções adquiridas no passado (BRASIL, 2014).

Foram registrados 430 casos da Doença de Chagas, somente no ano de 2006 no estado do Pará, todos os pacientes que foram notificados tinham ingerido açaí em determinados pontos de vendas (BRASIL, 2010a). Entre o ano de 2000 a 2011, foram notificados mais de mil e duzentos casos, sendo 70\% por transmissão oral, $7 \%$ por transmissão vetorial e $22 \%$ dos casos, não foi possível identificar o modo de transmissão (BRASIL, 2013a).

A estimativa é que existam entre dois e três milhões de pessoas infectadas. No entanto, nos últimos anos, a ocorrência de Doença de Chagas tem sido observada em diferentes Estados, em especial na região da Amazônia Legal, principalmente, em decorrência da transmissão oral (BRASIL, 2014a).

Outra doença preocupante no Brasil é a Raiva. O vírus da Raiva pertence ao gênero Lyssavirus, da família Rhabdoviridae. É uma doença transmitida ao homem pelo patógeno presente na saliva e secreções do animal infectado, principalmente pela mordedura. O morcego é o principal responsável pela manutenção da cadeia silvestre no Brasil, já o cão, em alguns municípios, permanece sendo fonte de contaminação em áreas urbanas (BRASIL, 2011b). 
Os casos de atendimentos notificados anti-rábicos em humanos têm aumentado nos últimos dez anos. Em 2000, foram registrados pelo menos 234 mil atendimentos, no ano de 2009, 440 mil ocorrências, aproximadamente $84 \%$ dos atendimentos tiveram exposição a animais domésticos (BABBONI \& MODOLO, 2011).

Desde a década de 80, o número de casos de raiva tem diminuído devido ao aumento do tratamento após exposição embora haja um número alto de casos de abandono pelos doentes, o paciente não imunizado compromete sua própria sobrevivência (VELOSO et al., 2011).

Dentre as diversas doenças, a Leishmaniose é uma doença causada por protozoários do gênero Leishmania, transmitidas ao homem por meio de vetores flebotomíneos infectados, possuindo grandes manifestações clínicas, a principal forma de transmissão do parasita para o homem e outros hospedeiros mamíferos é através da picada da fêmea (GONTIJO \& MELO, 2004).

A leishmaniose visceral americana (LVA) é uma doença que atinge seres humanos e outras espécies de animais silvestres e domésticos, causada pelo protozoário Leishmania chagasi, cujo principal vetor é o inseto Lutzomyia longipalpis (FIGUEIREDO et al., 2010).

A Leishmaniose Tegumentar Americana (LTA) é uma doença infecciosa, porém não contagiosa causada por diversas espécies de protozoários do gênero Leishmania, atinge principalmente a mucosa e pele, além disso, afeta diversos mamíferos domésticos, silvestres e humanos (BRASIL,2010b).

Dentre os casos de leishmaniose visceral registrados na América Latina, O Brasil é responsável por 90\% dos registros, sendo considerado o terceiro país com maior ocorrência no mundo (FURTADO et al., 2015). Segundo o mesmo autor, entre 1980 e 2008, foram registrados mais de 70 mil casos da doença no Brasil levando mais de 3.800 óbitos, além disso, o Maranhão é o estado com maiores números de casos registrados.

Outra zoonose preocupante no país é a Leptospirose, possui importância mundial, causada por bactérias do gênero Leptospira transmitidas pelo contato com urina de animais infectados ou água e lama contaminadas pela bactéria (BRASIL, 2014b,s/d).

O principal reservatório da Leptospira é o rato, principalmente nas áreas urbanas. A transmissão para o homem ocorre através de contato direto com sangue, tecidos, órgãos ou urina de animais infectados, ou por meio do contato indireto, quando a mucosa ou pele lesada entra em contato com água contaminada (DAHER et al., 2010).

Entre os casos registrados de leptospirose entre os anos de 2010 a 2014 foram confirmados 20.810 casos em todos os Estados da federação, 1.694 óbitos, com maior prevalência nas regiões sul e sudeste (BRASIL, 2016a). Os Estados com maior percentual de casos confirmados foram São Paulo (20,9\%), Santa Catarina (10,7\%), Rio Grande do Sul (10,6\%) e Acre (10,5\%), o que denota o importante trabalho a ser feito acerca do saneamento principalmente nas populações vulneráveis onde não há acesso a esses recursos (BRASIL, 2016a,b).

Doença re-emergente no Brasil, em algumas regiões tornou-se preocupação nacional por parte dos órgãos de saúde pública no país, a Febre Amarela é transmitida por um vírus pertencente ao gênero Flavivirus 
da família Flaviviridae (VASCONCELOS, 2002). É uma enfermidade infecciosa não contagiosa, que infecta o homem através da picada de insetos hematófagos (VASCONCELOS, 2002). A doença possui dois ciclos epidemiológicos distintos de transmissão: urbano, transmitidos pelos vetores Aedes aegypti e A. Albopictus,e o silvestre, pelos vetores Aedes, Sabethes e Haemagogus, culicídeos importantes no processo epidemiológico gerando alto risco de contaminação em áreas urbanas (BRASIL, 2017).

No Brasil a febre amarela é uma doença que ocorre principalmente na região amazônica, porém, surtos da doença são registrados de tempos em tempos. De 1982 a 2008, foram registrados 675 casos com 334 óbitos, um aumento de $49 \%$ dos casos registrados (BRASIL, 2009a).

A febre amarela estava erradicada do meio urbano brasileiro desde 1942 e sem números de casos significativos em áreas rurais desde 2009, porém a doença voltou a trazer preocupações para as autoridades sanitárias do Brasil (BEDINELLI, 2017).

Um suposto surto da doença no interior do estado de Minas Gerais pode ter sido a causa de 47 mortes, tendo em vista os impactos ambientais na região favorecendo o crescimento populacional dos vetores em detrimento de seus respectivos predadores, outro fator ponderaste ao aumento de casos foi a proximidade destes povoados com os grandes centros urbanos da região, a maioria dos envolvidos não eram vacinados contribuindo para a transmissão elevada da doença em detrimento da cobertura vacinal reduzida (ROMANO et al., 2011; BEDINELLI, 2017).

Em consonância com as demais doenças prevalentes no Brasil, a dengue é uma doença infecciosa causada por um vírus do gênero flavivírus, transmitida ao homem pelo mesmo vetor da febre amarela em ambientes urbanos (BRASIL, 2009b). O mosquito Aedes aegypti habita áreas urbanas, seus hábitos são diurnos e alimenta-se de sangue humano, o repasto sanguíneo ocorre ao amanhecer e entardecer podendo também picar a noite (BRASIL, 2008b).

Diferente do Aedes aegypti, o A. albopictus está presente nos ambientes urbanos, suburbano e rural, para se proliferar não depende de lugares com grande concentração pessoas devido a gama de hospedeiros, tem competência de colonizar os mais variados ambientes embora seu registro de maior prevalência esteja em áreas de cobertura vegetal preservada (AGUIAR, 2008).

Os primeiros registros do A. Albopictus no Brasil foi em 1986 no estado do Rio de Janeiro, no mesmo ano, foram encontrados nos estados de São Paulo e Minas Gerais e, no ano seguinte, no Espírito Santo (BRASIL, 2008c). Portanto, em um ano, A. albopictus estava alojado em todos os estados da região sudeste (CONSOLI \& OLIVEIRA, 1994).

No ano de 2016 o número de casos de dengue aumentou em comparação a 2015, até novembro foram notificados 1.475.940 casos de dengue no Brasil, considerando as regiões do país, o sudeste com 852.144, nordeste com 322.322, regiões centro-oeste com 190.623, sul 72.908 e norte 37.943 casos notificados (BRASIL, 2016b). Atualmente, a dengue está presente em quase todos os países do mundo, sobretudo na zona tropical.

Assim como a dengue, a malária é uma das doenças mais preocupantes do mundo. Embora seja causada por protozoários do gênero Plasmodium, a doença é de grande impacto em mais de 100 países, 
colocando em risco $40 \%$ da população mundial, são aproximadamente 2,4 bilhões de pessoas infectadas (GOMES et al., 2011).

O Brasil é o país que concentra o maior número de casos notificados do continente americano, a estimativa é de mais de 300.000 casos anuais (BRASIL, 2010c; GOMES et al., 2011). O quadro epidemiológico de malária é preocupante nos dias atuais, apesar de ter diminuído, o número de casos notificados no ano de 2008 foi 300.000, sendo que 99,9\% deles foram registrados na Amazônia Legal (BRASIL, 2010c).

Transmissões de doenças vetoriais são responsáveis por um elevado número de mortalidade principalmente em países mais pobres, causadas pela carência escolar e pobreza, sobrecarregando o sistema de saúde (BRASIL, 2014c).

No Brasil, depois da dengue, as doenças que mais preocupam são a leishmaniose visceral e a malária, essas doenças têm diferentes formas dos vetores se adaptarem ao clima (BRASIL,2010b; 2014c). A alteração climática que vem acontecendo nos últimos tempos, tem cada vez maior influência na transmissão de doenças infecciosas, especialmente as transmitidas por vetores, as quais ainda continuam sendo uma causa importante de mortalidade no Brasil e no mundo (BARCELLOS et al., 2009).

Diante do grande número de fatores socioambientais e climáticos que influencia na proliferação de doenças, fica claro que é preciso realizar ações efetivas para que estes fatores não venham prejudicar a saúde e o bem-estar da população dentre essas medidas está o controle vetorial em áreas de risco, atividades de educação ambiental e saúde e a correta distribuição e acesso ao saneamento básico (RIBEIRO, 2004).

\section{Saneamento no Brasil: Acesso e Distribuição Nacional}

Segundo a Organização Mundial de Saúde (2007), saneamento básico é a influência de todos os fatores do meio físico do homem, que desempenham efeitos sobre o bem-estar mental, social e físico. 0 início do saneamento no Brasil aconteceu em 1561, quando Estácio de Sá mandou cavar o primeiro poço para abastecer a cidade do Rio de Janeiro. Em 1673, deu-se início, e ficou pronto em 1723, conduzindo águas do rio Carioca em direção ao Chafariz (BARROS, 2014).

O Programa de Saneamento Básico, cuja execução está no desempenho da Fundação Nacional de Saúde (FUNASA), foi criado com meta inicial de fornecer saneamento básico aos municípios com população abaixo de 30.000 habitantes, e assim promover condição ambiental (BRASIL, 2002a). O saneamento básico é importante para a população em geral, é um direito de todos e essas ações estão vinculadas a dignidade da humanidade, que envolve toda uma questão cultural, ambiental e sanitária (SAKER, 2007).

O Brasil é uma terra privilegiada quanto ao volume de recursos hídricos, possuindo $13,7 \%$ da água doce do mundo (LIMA \& SILVA, 2007). Contudo, segundo o mesmo autor, a disponibilidade desses recursos não é igual, $73 \%$ da água doce no país está na bacia Amazônica, que é habitada por menos de $5 \%$ da população. Somente $\mathbf{2 7 \%}$ dos recursos hídricos brasileiros estão disponíveis para as outras regiões, de onde habitam 95\% da população do país, por outro lado, a ausência de planejamento e instrumentos relativos a saúde pública constitui uma lacuna nos programas de saneamento brasileiro (HELLER, 1997; LIMA \& SILVA, 2007) 
O Sistema de Fornecimento de Água representa o conjunto de obra, abastecimentos e serviços reservados ao fornecimento de água potável de uma sociedade para o consumo de serviços públicos, doméstico, consumo industrial e outros usos (BARROS, 1995).

Problemas relacionados à saúde pública e a poluição do ambiente faz com que a humanidade encontre soluções para resolverem os problemas de saneamento básico como tratamento de esgoto, coleta de lixo e o tratamento dos resíduos sólidos para assim ter um abastecimento de água seguro para o consumo humano (RIBEIRO \& ROOKE, 2010). O objetivo do saneamento é controlar e prevenir doenças, proporcionando uma melhoria na qualidade de vida da população (SOUZA et al., 2010).

A contaminação das águas naturais representa alto risco a saúde humana, essa estreita relação entre a qualidade e inúmeras enfermidades nas populações humanas está ligada por serviços de saneamento prestados, a causalidade entre essas condições e o quadro epidemiológico é discutido pela lei 8.080/90, citando sobre os fatores determinantes na prestação de serviços em saúde (LIBÂNIO et al., 2005).

\section{Principais Problemas Associados a Zoonoses e Saneamento}

A Constituição Federal definiu que a saúde é direito de todos e dever do Estado e a Lei Federal $\mathrm{n}$. 8.080/1990, que regulamentou o SUS, prevê em seu Artigo 7ํ, como princípios do sistema, entre outros (MOURA, 2017). O Sistema Único de Saúde (SUS) garante que todas as pessoas tenham direito as ações de promoção, proteção e recuperação da saúde oferecida pela a atenção básica (BRASIL, 2006b).

As ações humanas no ambiente como a urbanização, pecuária e industrialização vêm proporcionando uns maiores contatos entre seres humanos e animais silvestres e domésticos, facilitando assim a proliferação de agentes infecciosos, esses animais tanto em vida livre como em cativeiro podem ser portadores ou reservatórios de doenças (BARBOSA et al., 2011).

As doenças infecciosas são abundantes, desenvolvidas na maioria das vezes por agravos ao ambiente, são causadas por agentes etiológicos vivos, parasitas adquiridos em um determinado período pelos hospedeiros a partir do ambiente contaminado (PIGNATTI, 2004). As zoonoses são tipicamente endêmicas e acontecem através de focos naturais, porém mudanças no ecossistema e eventos climáticos podem causar epidemias em um determinado espaço geográfico (SERAFIM, 2013). Além disso, um aumento expressivo do número de animais de estimação nos domicílios e animais sinantrópicos tem contribuído para a disseminação de doenças (PREGER, 2002). A falta de saneamento básico aliado as chuvas, enchentes, lixos urbanos, e a alta densidade demográfica geram grandes contingentes propícios a tais endemias (TASSINARI et al., 2004; PREGER, 2002).

A interface do saneamento ambiental atua como um fundo cobertor para que os serviços em saúde sejam de fato aplicados, a evitabilidade de doenças nas populações vulneráveis bem como todos os processos de cobertura econômica, social, cultural e ambiental transpassa a gestão de recursos hídricos, saneamento e saúde pública disponíveis para todos. Esses serviços essenciais (figura 1) é a condição importante para garantir o bem-estar social que pode ser apreendido pelo alcance do abastecimento de água e esgotamento sanitário (LIBÂNIO et al., 2005). 
O objetivo da vigilância epidemiológica é prevenir, eliminar, controlar, erradicar as doenças e evitar caso de óbitos e sequelas. Nos últimos anos, têm-se verificado intensas alterações no perfil epidemiológico da população brasileira, com as baixas taxas de mortalidade por doenças parasitárias e infecciosas (BRASIL, 2002b).

A Vigilância Epidemiológica instituiu um modelo padrão de acompanhamento de doenças infecciosas, ampliado um conjunto de episódios que envolvem desde as más formações congênitas até as enfermidades crônico-degenerativas; este órgão vem, pouco a pouco, sendo empregado pelos órgãos de Vigilância Sanitária (ROZENFELD, 2000).

As atividades da Vigilância Epidemiológica são constantes, envolvendo todo o grupo, apesar dos papéis serem diferentes, todos têm a responsabilidade para vigiar e zelar pela saúde da população (CERQUEIRA, 2003; BRASIL, 2013b).

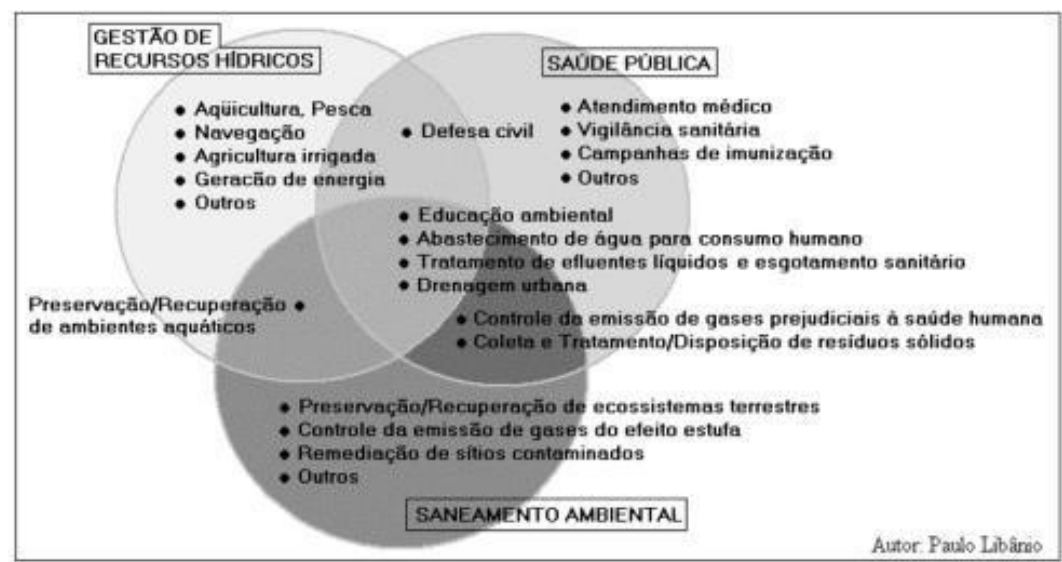

Figura 1: Interfaces do saneamento ambiental com a gestão de recursos hídricos e com a saúde pública. Fonte: Libânio et al., (2005).

\section{Desafios e perspectivas na saúde pública brasileira}

As doenças emergentes ou re-emergentes são, simultaneamente, enfermidades novas e exóticas, aparecem após um período de decadência expressivo ou com risco de aumento no futuro próximo promovendo impacto sobre o ser humano, devido à sua gravidade, podem deixar sequelas e até levar à morte (BRASIL, 2016b). Essas doenças podem envolver uma ou mais espécies de animais no seu ciclo de transmissão, trazida para o Brasil por meio da entrada de pessoa e animal infectado ou ser veiculado pela ação do ar, água e solo (BRASIL, 2016a; SOARES et al., 2002; PIGNATTI, 2004).

Nos últimos anos, tem-se notado episódios de novas enfermidades, bem como o reaparecimento de doenças datadas como erradicadas (CARVALHO et al., 2009). A degradação socioecológica, resultante de processos de transformação social orientados por modelos econômicos, revelou-se como responsável pela disseminação de novos agentes etiológicos e como determinante de mudanças do padrão epidemiológico de doenças (MINAYO \& MIRANDA, 2002).

A medida de prevenção voltada para a saúde população tem como objetivo evitar que os indivíduos saudáveis venham adoecer, e atender de forma rápida aqueles que adoeceram, essas ações são realizadas 
pelo o serviço de saúde através de campanhas como o programa de imunização e o programa de saúde da família (BRASIL, 2010a).

As mudanças da população nos últimos anos, nos quais ressalta o declínio das taxas de mortalidade por doenças infecciosas e parasitárias, a vigilância epidemiológica vem orientar a população a executar ações de controle para evitar agravos das doenças, essas ações devem ser contínuas (BRASIL, 2008a).

A educação em saúde ambiental contribui na conscientização e na cidadania estimulando a participação da população. As ações devem ser vivenciadas e compartilhadas pela a equipe do SUS e usuários (BRASIL, 2012). Atualmente as questões ambientais é um problema de saúde, o desenvolvimento econômico contribuiu para a minoração da preocupação ambiental, sendo responsáveis pelos danos causados aos sistemas ecológicos, afetando os ambientes naturais e urbanos (BESERRA et al., 2010).

Para que a Educação Ambiental exerça sua função, não pode debater exclusivamente o meio ambiente físico, mas também deve abordar a realidade local e todas as relações entre o ambiente e sociedade. Dessa forma, deve ser destacado a saúde local, e os cuidados com a prevenção de doenças e fatores ambientais (PEREIRA et al., 2012).

A Educação Ambiental pode ser percebida como uma ação que procura desenvolver consciência e preocupação com o meio e com os problemas que existem. As pessoas que participam dessa transformação são consideradas agentes de transformação social, envolvidas na melhoria da qualidade de vida (TASSARA \& ARDANS, 2005).

\section{CONCLUSÃO}

A qualidade da assistência à saúde e seus recursos devem estar disponíveis para a sociedade, para isto, a importância dos serviços de saneamento básico, a prevenção de doenças, a preservação do ambiente, as ações de saneamento deverão ser prioridade para o poder público. Porém é necessário que tenha um equilíbrio entre os aspectos ecológicos, econômicos e sociais de tal forma que as necessidades básicas de cada cidadão possam ser satisfeitas, sem consumismo ou desperdícios, que todos tenham oportunidades iguais de desenvolvimento de seus próprios potenciais e tenham consciência de preservação dos recursos naturais e na prevenção de doenças.

Concluiu-se que o saneamento básico é fundamental para evitar doenças e oferecer melhor condição de vida para a população. Além disso, a efetividade dos programas de educação ambiental na prevenção de doenças, (re)educação das populações vulneráveis através de novos saberes pode ser um instrumento eficaz para a minimização dos impactos ocasionados pela ação antrópica aliado aos programas de saúde e segurança pública.

\section{REFERÊNCIAS}

AGUIAR, D. B.; FONTÃO, A.; RUFINO, P.; MACEDO, V. A.; VELÁSQUEZ , C. M. R.; CASTRO, M. G.; HONÓRIO, N. A.. Primeiro registro de Aedes albopictus (Diptera: Culicidae) em Roraima, Brasil. Acta Amazonica, v.38, n.2, p.357-360, 2008.
BABBONI, S. D.; MODOLO, J. R.. Raiva: origem, importância e aspectos históricos. UNOPAR científica: Ciências Biológicas e da Saúde, p.349-356, 2011. 
BARBOSA, A. D.; MARTINS, N. R. S.; MAGALHÃES, D. F. Zoonoses e saúde pública: riscos da proximidade humana com a fauna silvestre. Ciência Veterinária nos Trópicos, v.14, n.1/2/3, p.1-9, 2011.

BARCELLOS, C.; MONTEIRO, A, M, V.; CORVALAN, C. Mudanças climáticas e ambientais e as doenças infecciosas: cenários e incertezas para o Brasil. Epidemiol. Serv. Saúde, v.18, n.3, 2009.

BARROS, R. T. V. Saneamento: manual de saneamento e proteção ambiental para os municípios. v 2. Belo Horizonte: Escola de Engenharia da UFMG, 1995.

\section{BARROS, R. A.. História do saneamento básico no Brasil,} 2014.

BEDINELLI, T.. Casos de febre amarela aumentam em Minas Gerais e geram apreensão Minas Gerais. 2017.

BESERRA, E. P.. Educação ambiental e enfermagem: uma integração necessária. Rev. Bras. Enferm., v.63, n.5, p.848$52,2010$.

BRASIL. Fundação nacional de saúde. Guia de vigilância epidemiológica. 5 ed. Brasília: FUNASA, 2002a.

BRASIL. Ministério da Saúde. Programa Saneamento Básico. Brasília: Ministério da Saúde, 2002b.

BRASIL. Fundação Nacional de Saúde. Manual de saneamento. 3 ed. Brasília: FUNASA, 2006a.

BRASIL. Ministério da Saúde. Carta dos direitos dos usuários da saúde. Brasília, DF, 2006b.

BRASIL. Ministério da Saúde. Secretaria de Atenção à Saúde. Departamento de Atenção Básica. Vigilância em Saúde: Dengue, Esquistossomose, Hanseníase, Malária, Tracoma e. 2 ed. Brasília: Ministério da Saúde, 2008a.

BRASIL. Conheça o comportamento do mosquito Aedes aegypti e entenda a razão que leva este pequeno inseto a ser taxado desta forma. 2008b.

BRASIL. Vetor da dengue na Ásia, A. albopictus é alvo de estudos. Rio de Janeiro: Instituto Oswaldo Cruz/FIOCRUZ, 2008c.

BRASIL. Ministério da Saúde. Secretaria de Atenção à Saúde. Departamento de Atenção Básica. Vigilância em saúde: zoonoses. Brasília: Ministério da Saúde, 2009a.

BRASIL. 0 agente comunitário de saúde no controle da dengue. Brasília: Ministério da Saúde, 2009b.

BRASIL. Açaí contaminado com parasito pode transmitir doença de Chagas. 2010a.

BRASIL. Ministério da Saúde. Secretaria de Vigilância em Saúde. Manual de Vigilância da Leishmaniose Tegumentar Americana. 2 ed. Brasília: Ministério da Saúde, 2010b.

BRASIL. Módulos de Princípios de Epidemiologia para o Controle de Enfermidades. Módulo 6: controle de enfermidades na população. Brasília: Organização PanAmericana da Saúde; Ministério da Saúde, 2010c.

BRASIL. Fundação Nacional de Saúde. Saneamento para promoção da saúde. FUNASA, Brasil, 2011a.
BRASIL. Ministério da Saúde. Secretaria de Vigilância em Saúde. Departamento de Vigilância Epidemiológica. Normas técnicas de profilaxia da raiva humana. Brasília: Ministério da Saúde, 2011b.

BRASIL. Educação em saúde ambiental a FUNASA, 2012.

BRASIL. Doença de Chagas. Rio de Janeiro: Fundação Oswaldo Cruz; Ministério da Saúde, 2013a.

BRASIL. Secretaria de Estado da Saúde de São Paulo. Centro de Vigilância Epidemiológica Prof. Alexandre Vranjac. Caderno de vigilância epidemiológica: vigilância epidemiológica em saúde ambiental. São Paulo, 2013b.

BRASIL. Ministério da saúde. Doença de Chagas, 2014a.

BRASIL. Ministério da Saúde. Secretaria de Vigilância em Saúde. Departamento de Vigilância das Doenças Transmissíveis. Leptospirose: diagnóstico e manejo clínico. Brasília: Ministério da Saúde, 2014b.

BRASIL. OMS alerta para doenças transmitidas por vetores e Ensp lista as mais comuns no Brasil, 2014c.

BRASIL. Ministério da Saúde. Secretaria de Vigilância em Saúde. Departamento de Vigilância das Doenças Transmissíveis. Manual de vigilância, prevenção e controle de zoonoses. Brasília: Ministério da Saúde, 2016a.

BRASIL. Monitoramento dos casos de Dengue, Chikungunya e Zika. Brasília: 2016b.

BRASIL. Ministério da Saúde. Febre Amarela. 2017.

BRASIL. Ministério da Saúde. Situação Epidemiológica. 2017.

CARVALHO, J. A.. Doenças emergentes: uma análise sobre a relação do homem com o seu ambiente. Revista Práxis, v.1, n.1, 2009.

CERQUEIRA, E. M.. Vigilância Epidemiológica no processo de municipalização do Sistema de Saúde em Feira de SantanaBA. Revista Epidemiologia e Serviços de Saúde, Brasília, v.12, n.4, 2003

CONSOLI, R. A. G. B.; OLIVEIRA, R. L.. Principais mosquitos de importância sanitária no Brasil. Rio de Janeiro: FIOCRUZ, 1994

DAHER, E. F.; SILVA JUNIOR, G. B.; ABREU, K. L. S Insuficiência renal aguda associada à leptospirose. J. bras. nefrol, v.32, n.4, p.408-415, 2010.

FIGUEIREDO, F. B. et al. Relato de caso autóctone de leishmaniose visceral canina na zona sul do município do Rio de Janeiro. Ver. Soc. Bras. Med. Trop., v.43, n.1, p.98-99, 2010.

FRANCEYS, R.; PICKFORD, J.; REIED, R.. Guia para el desarollo del saneamiento in situ. OMS, 1994.

FURTADO, A.. Análise espaço-temporal da leishmaniose visceral no estado do Maranhão, Brasil. Revista Ciência \& Saúde Coletiva, v.20, n.12, 2015.

GIL, A. C.. Como elaborar projetos de pesquisa. 4 ed. São Paulo: Atlas, 2002. 
GOMES, K. B.. Giardia duodenalis: genotypic comparison between a human and a canine isolates. Revista da Sociedade Brasileira de Medicina Tropical, v.44, n.4, 2011.

GONTIJO, C. M. F.; MELO, M. N.. Leishmaniose visceral no Brasil: quadro atual, desafios e perspectivas. Ver. Bras. Epidemiol., p.338-349, 2004.

HELLER, L.. Saneamento e Saúde. Organização Panamericana de Saúde. Brasília: OMS, 1997.

LEONETI, A. B.. Saneamento básico no Brasil: considerações sobre investimentos e sustentabilidade para o século XXI. Revista de Administração Pública, v.45, n.2, p.331-348, 2011.

LIBÂNIO, P. A. C.; CHERNICHARO, C. A. L.; NASCIMENTO, N. O.. A dimensão da qualidade de água: avaliação da relação entre indicadores sociais, de disponibilidade hídrica, de saneamento e de saúde pública. Engenharia Sanitária e Ambiental, v.10, n.3, p.219-228, 2005.

LIMA, A. L. G. S.; PINTO, M. M. S.. Fontes para a história dos 50 anos do Ministério da Saúde. Hist. Cienc. Saúde, Manguinhos, v.10, n.3, p.1037-1051, 2003.

LIMA, J. E. F. W.; SILVA, E. M.. Gestão de recursos hídricos e manejo da irrigação no Cerrado. In: FALEIRO, F. G.; SOUSA, E. S.. Pesquisa, desenvolvimento e inovação para o Cerrado. Planaltina: Embrapa Cerrados, 2007.

LIMA, M. C. F.. Principais zoonoses em pequenos animais: breve revisão. Veterinária e Zootecnia, v.24, n.1, p.84-106, 2017.

MINAYO, M. C. S.; MIRANDA, A. C.. Saúde e ambiente sustentável: estreitando nós. Rio de Janeiro: FIOCRUZ, 2002.

MOURA, E S. O direito à saúde na Constituição Federal de 1988. Rio Grande, 2017.

OMS. Organização Mundial da Saúde. Zoonoses. 2017.

PEREIRA, C. A. R.; MELO, J. V.; FERNANDES, A. L. T. A educação ambiental como estratégia da Atenção Primária à Saúde. Revista Brasileira de Medicina de Família e Comunidade, v.7, n.23, p.108-116, 2012.

PIGNATTI, M. G.. Saúde e ambiente: as doenças emergentes no Brasil. Ambiente \& Sociedade, v.7, n.1, p.133-144, 2004.

PREGER, J.. Animais de Estimação: da competição à simbiose. Monografia (Residência Médica em Psiquiatria) Hospital Psiquiátrico São Pedro, Porto Alegre, 2002.

RIBEIRO, H.. Saúde Pública e meio ambiente: evolução do conhecimento e da prática, alguns aspectos éticos. Saúde e Sociedade, v.13, n.1, 2004.

RIBEIRO, C. T. M.. O sistema público de saúde e as ações de reabilitação no Brasil. Ver. Panam. Salud Publica, v.28, n.1, p.43, 2010.

RIBEIRO, J. W.; ROOKE, J. M. S.. Saneamento básico e sua relação com o meio ambiente e a saúde pública.
Monografia (Especialização em Análise Ambiental) Universidade Juiz de Fora, Juiz de Fora, 2010.

ROMANO, A. P. M.. Febre amarela no Brasil: recomendações para a vigilância, prevenção e controle. Epidemiologia e Serviços de Saúde, v.20, n.1, 2011.

ROZENFELD, S.. Fundamentos da vigilância sanitária. In: Fundamentos da vigilância sanitária. Rio de Janeiro: FIOCRUZ, 2000.

SAKER, J. P. P.. Saneamento Básico e Desenvolvimento. Dissertação (Mestrado em Direito Político e Econômico) Universidade Presbiteriana Mackenzie, São Paulo, 2007.

SANGUINETTE, S. P.. Zoonoses em livros didáticos de ciências: uma contribuição entre às articulações entre educação e saúde. Monografia (Licenciatura em Biologia) Universidade Federal do Rio Grande do Sul, 2015.

SERAFIM, M. L. Identificação e perfil de resistência a antimicrobianos de bactérias isoladas de diferentes amostras provenientes do aterro controlado da cidade de Campos dos Goytacazes-RJ. Dissertação (Mestrado em Ciência Animal) - Universidade Estadual do Norte Fluminense, 2013.

SILVEIRA, A. C.; PIMENTA JUNIOR, F.. A inserção institucional do controle da doença de Chagas. Rev. Soc. Bras. Med. Trop., v.44, n.2, p.19-24, 2011.

SOARES, S. R. A.; BERNARDES, R. S.; NETTO, O. M. C. Relações entre saneamento, saúde pública e meio ambiente: elementos para formulação de um modelo de planejamento em saneamento. Cad. Saúde Pública, v.18, n.6, p.1713-1724, 2002.

SOUZA, R. S.. Saneamento básico no estado de Roraima: situação atual e perspectivas. Análise: Revista de Administração da PUCRS, v.21, n.2, 2010.

TASSARA, E. T. O.; ARDANS, O.. Intervenção psicossocial: desvendando o sujeito histórico e desvelando os fundamentos da educação ambiental crítica. In: FERRARO JÚNIOR, L.A.. Encontros e Caminhos: formação de educadoras (es) ambientais e coletivos educadores. Brasília: MMA, 2005.

TASSINARI, W. S.. Distribuição espacial da leptospirose no Município do Rio de Janeiro, Brasil, ao longo dos anos de 1996-1999. Cad. Saúde Pública, v.20, n.6, p.1721-1729, 2004.

TEIXEIRA, J. C.; GUILHERMINO, R. L.. Análise da associação entre saneamento e saúde nos estados brasileiros, empregando dados secundários do banco de dados indicadores e dados básicos para a saúde 2003-IDB 2003. Eng Sanit Ambient, v.11, n.3, p.277-282, 2006.

VASCONCELOS, P. F. C.. Febre amarela: reflexões sobre a doença, as perspectivas para o século XXI e o risco da reurbanização. Rev. Bras. Epidemiol., Belém, v.5, n.3, 2002.

VELOSO, R.. Motivos de abandono do tratamento antirrábico humano pós-exposição em Porto Alegre (RS, Brasil). Ciência \& Saúde Coletiva, v.16, n.2, 2011. 